\title{
An Iteration Scheme Suitable for Solving Limit Cycles of Nonsmooth Dynamical Systems
}

\author{
Q. X. Liu, Y. M. Chen, and J. K. Liu \\ Department of Mechanics, Sun Yat-sen University, Guangzhou 510275, China \\ Correspondence should be addressed to Y. M. Chen; chenyanmao@hotmail.com
}

Received 19 July 2013; Accepted 26 September 2013

Academic Editor: Mufid Abudiab

Copyright (C) 2013 Q. X. Liu et al. This is an open access article distributed under the Creative Commons Attribution License, which permits unrestricted use, distribution, and reproduction in any medium, provided the original work is properly cited.

\begin{abstract}
The Mickens iteration method (MIM) is modified to solve self-excited systems containing nonsmooth nonlinearities and/or nonlinear damping terms. If the MIM is implemented routinely, the unknown frequency and amplitude of limit cycle (LC) would couple to each other in complicated nonlinear algebraic equations at each iteration. It is cumbersome to solve these algebraic equations, especially for nonsmooth systems. In the modified procedures, the unknown frequency is substituted by the determined value obtained at the previous iteration. By this means, the frequency is decoupled from the nonlinear terms. Numerical examples show that the LCs obtained by the modified MIM agree well with numerical results. The presented method is very suitable for solving self-excited systems, especially those with nonlinear damping and nonsmooth nonlinearities.
\end{abstract}

\section{Introduction}

Recent years have witnessed the wide applications of iteration techniques, such as the Mickens iteration method (MIM) [1-6] and the variational iteration method [7-10]. In order to improve the efficiency, Lim and Wu [11], Marinca and Herisanu [12], and Hu [13] modified the MIM, respectively.

In principle, the approximations can be obtained to any desired accuracy by the MIM as long as the iteration proceeds. In the MIM, algebraic equations are introduced at each iteration to eliminate the so-called secular terms. In the applications in conservative oscillators, the algebraic equations are linear. Nonlinear damping and nonsmooth terms appear widely in many dynamical systems $[14,15]$. As for the oscillators with nonlinear damping terms, however, very complicated nonlinear algebraic equations have to be solved at each iteration [16]. Moreover, the algebraic equations cannot be deduced for systems with nonsmooth nonlinearities. It is necessary and worthwhile, therefore, to propose some approaches to simplify the MIM. This paper will present a modified iteration algorithm by decoupling the unknown frequency from nonlinear terms.

\section{A Modified MIM}

Consider a self-excited oscillator

$$
\ddot{x}+f(x, \dot{x})=0,
$$

where the superscript denotes the differentiation with respect to time $t$ and $f(x, \dot{x})$ is a nonlinear term with damping terms. Assume that system (1) has at least one limit cycle (LC) solution. Since the LC frequency and amplitude are independent of initial conditions, they should be considered as unknowns to be determined at every iteration. Denote the angular frequency as $\omega$ and introduce the transformation as $\tau=\omega t$; thus, we rewrite (1) as

$$
\omega^{2} x^{\prime \prime}+\omega^{2} x=\omega^{2} x-f\left(x, \omega x^{\prime}\right)
$$

subject to the following initial conditions:

$$
x(0)=\alpha, \quad x^{\prime}(0)=0,
$$

where $\alpha$ is the unknown LC amplitude and the superscript denotes the differentiation with respect to $\tau$. Note that $\alpha$ will be approximated as a series $\left\{\alpha_{k}\right\}$ by eliminating the secular 
terms at each iteration stage. In order to obtain the LC, the MIM [16] can be given as

$$
\begin{array}{r}
\omega_{k-1}^{2}\left(x_{k}^{\prime \prime}+x_{k}\right)=\omega_{k-1}^{2} x_{k-1}-f\left(x_{k-1}, \omega_{k-1} x_{k-1}^{\prime}\right), \\
k=1,2, \ldots,
\end{array}
$$

with the initial conditions being rewritten at each iteration as

$$
x_{k}(0)=\alpha_{k}, \quad x_{k}^{\prime}(0)=0 .
$$

Note that the coefficient of the first harmonic in $x_{k-1}$ remains still to be an unknown, that is, $\alpha_{k-1}$. This unknown will couple with the unknown frequency, $\omega_{k-1}$, which will result in a coupled nonlinear term (i.e., $\omega_{k-1} x_{k-1}^{\prime}$ ) in the right side of (4). If higher powers of $x^{\prime}$ exist, these terms will lead to very complicated functions in $\omega_{k-1}$ and $\alpha_{k-1}$. Different from conservative systems, $\omega_{k-1}^{2}$ can no longer be considered as an independent unknown. In order to simplify the MIM, therefore, a modified scheme is proposed as

$$
\begin{array}{r}
\omega_{k-1}^{2}\left(x_{k}^{\prime \prime}+x_{k}\right)=\omega_{k-1}^{2} x_{k-1}-f\left(x_{k-1}, \omega_{k-2} x_{k-1}^{\prime}\right), \\
k=1,2, \ldots .
\end{array}
$$

As $k=1$, we choose $\omega_{-1}=\omega_{0}$. In the $k$ th iteration, $\omega_{k-2}$ is a given constant that is obtained at the $(k-1)$ th iteration. The square of the unknown frequency, that is, $\omega_{k-1}^{2}$, can be treated as an independent parameter, because $\omega_{k-1}$ appears only in $\omega_{k-1}^{2} x_{k-1}$. According to the initial conditions, the starting iteration solution can be chosen as

$$
x_{0}(\tau)=\alpha_{0} \cos \tau \text {. }
$$

It is obvious that as long as the series $\left\{\omega_{k}, k=1,2, \ldots\right\}$ and $\left\{x_{k}, k=1,2, \ldots\right\}$ are convergent, they must converge to the exact solutions. The right-hand side of (6) can be expressed by Fourier series as

$$
\begin{aligned}
\omega_{k-1}^{2} x_{k-1}-f\left(x_{k-1}, \omega_{k-2} x_{k-1}^{\prime}\right) \\
=\sum_{i=1}^{\varphi(k)}\left[c_{k-1, i}\left(\omega_{k-1}^{2}, \alpha_{k-1}\right) \cos (i \tau)\right. \\
\left.\quad+s_{k-1, i}\left(\omega_{k-1}^{2}, \alpha_{k-1}\right) \sin (i \tau)\right],
\end{aligned}
$$

where the harmonic coefficients $c_{k-1, i}\left(\omega_{k-1}^{2}, \alpha_{k-1}\right)$ and $s_{k-1, i}\left(\omega_{k-1}^{2}, \alpha_{k-1}\right)$ are functions in $\omega_{k-1}^{2}$ and $\alpha_{k-1}$. Here, $\varphi(k)$ is a positive integer denoting the order of the highest harmonic. Approximations $\omega_{k-1}$ and $\alpha_{k-1}$ are determined by eliminating the secular terms, that is, letting

$$
\begin{array}{r}
c_{k-1,1}\left(\omega_{k-1}^{2}, \alpha_{k-1}\right)=0, \quad s_{k-1,1}\left(\omega_{k-1}^{2}, \alpha_{k-1}\right)=0, \\
k=1,2, \ldots .
\end{array}
$$

These equations can be solved analytically if $\omega_{k-1}^{2} \alpha_{k-1}$ is considered as an independent unknown. They can also be numerically solved by Newton-Raphson method. The latter is employed in this study.
Different from the existing procedures [16], as $k$ increases, $\omega_{k-1}^{2}$ is always an independent unknown in the modified MIM. Moreover, unknown $\omega_{k-1}$ does not couple with nonlinear terms. It simplifies the MIM to a large extent, as shown later.

\section{Numerical Examples}

Example 1 (system with nonlinear damping terms). The van der Pol equation is chosen to illustrate the previous procedures more clearly:

$$
\ddot{x}+x+\varepsilon\left(x^{2}-1\right) \dot{x}=0,
$$

where $\varepsilon$ is a given constant. As known, (10) has a stable LC solution when $\varepsilon>0$ while an unstable one when $\varepsilon<0$.

According to the modified MIM, the corresponding iteration scheme is given as

$$
\begin{array}{r}
\ddot{x}_{k}+\omega_{k-1}^{2} x_{k}=\omega_{k-1}^{2} x_{k-1}-x_{k-1}-\varepsilon\left(x_{k-1}^{2}-1\right) \dot{x}_{k}, \\
k=1,2, \ldots .
\end{array}
$$

Introducing a new time variable $\tau=\omega_{k-1} t$ at each iteration stage, we rewrite (11) as

$$
\omega_{k-1}^{2}\left(x_{k}^{\prime \prime}+x_{k}\right)=\omega_{k-1}^{2} x_{k-1}-x_{k-1}-\varepsilon \omega_{k-1}\left(x_{k-1}^{2}-1\right) x_{k-1}^{\prime},
$$

where the superscript denotes the derivative with respect to $\tau$. The iteration algorithm begins with an initial solution

$$
x_{0}(\tau)=\alpha_{0} \cos \tau
$$

Then, we obtain the governing equations in $x_{1}(\tau)$ as

$$
\begin{aligned}
\omega_{0}^{2}\left(x_{1}^{\prime \prime}+x_{1}\right)= & \left(\omega_{0}^{2} \alpha_{0}-\alpha_{0}\right) \cos \tau-\left(\varepsilon \alpha_{0} \omega_{0}-\varepsilon \frac{\alpha_{0}^{3} \omega_{0}}{4}\right) \sin \tau \\
& +\varepsilon \frac{\alpha_{0}^{3} \omega_{0}}{4} \sin 3 \tau, \quad x_{1}(0)=\alpha_{1}, \quad x_{1}^{\prime}(0)=0 .
\end{aligned}
$$

Equating the coefficients of $\cos \tau$ and $\sin \tau$ to zeros results into

$$
\omega_{0}^{2} \alpha_{0}-\alpha_{0}=0, \quad \varepsilon \alpha_{0} \omega_{0}-\varepsilon \frac{\alpha_{0}^{3} \omega_{0}}{4}=0
$$

which yields that $\omega_{0}=1$ and $\alpha_{0}=2$. Substituting them into (16), we have

$$
x_{1}^{\prime \prime}+x_{1}=2 \varepsilon \sin 3 \tau .
$$

Considering initial conditions (14), we can obtain

$$
x_{1}=\alpha_{1} \cos \tau+\frac{3 \varepsilon \sin \tau}{4}-\frac{\varepsilon \sin 3 \tau}{4},
$$

where $\alpha_{1}$ is to be determined at the next iteration stage. 
TABLE 1: Comparison of the second-order frequency obtained by IS and LP method with the forth-order approximation obtained by LP method, when $\varepsilon=1$.

\begin{tabular}{lccccc}
\hline$\varepsilon$ & $\omega_{2}^{\mathrm{IS}}$ & $\omega_{2}^{\mathrm{LP}}$ & $\omega_{4}^{\mathrm{LP}}$ & $\left|\omega_{2}^{\mathrm{IS}}-\omega_{4}^{\mathrm{LP}}\right|$ & $1.76 E-3$ \\
\hline 1 & 0.944799584 & 0.93750000 & 0.943033854 & $1.00 E-4$ & $5.53 E-3$ \\
0.5 & 0.984820946 & 0.98437500 & 0.984720866 & 0.996115367 & $6.09 E-6$ \\
0.25 & 0.996121460 & 0.99609375 & $0.96 E-4$ \\
\hline
\end{tabular}

According to iterative scheme (11), the equation in $x_{2}(\tau)$ is deduced as

$$
\begin{aligned}
\omega_{1}^{2}\left(x_{2}^{\prime \prime}+x_{2}\right)=\sum_{i=1}^{9}[ & c_{1, i}\left(\omega_{1}^{2}, \alpha_{1}\right) \cos \left(i \omega_{1} t\right) \\
& \left.+s_{1, i}\left(\omega_{1}^{2}, \alpha_{1}\right) \sin \left(i \omega_{1} t\right)\right] .
\end{aligned}
$$

Equate the coefficients of $\cos \tau$ and $\sin \tau$ to zeros:

$$
\begin{aligned}
& \frac{3 \omega_{1}^{2}}{4}-\frac{15 \alpha_{1}}{64}-\frac{\alpha_{1}^{3}}{4}-\frac{3}{4}=0, \\
& \alpha_{1} \omega_{1}^{2}-\alpha_{1}-\frac{\alpha_{1}^{3}}{8}+\frac{75}{128}=0 .
\end{aligned}
$$

By solving (19) numerically, we can determine $\alpha_{1}$ and $\omega_{1}^{2}$. Here, we obtain the second-order approximation and expand it as

$$
\omega_{2}^{\mathrm{IS}}=1-\frac{\varepsilon^{2}}{16}+\frac{29 \varepsilon^{4}}{2048}+o\left(\varepsilon^{4}\right) .
$$

According to [17], the Lindstedt-Poincare (LP) method provides the second- and forth-order approximate frequency $\omega_{2}^{\mathrm{LP}}=1-\varepsilon^{2} / 16$ and $\omega_{4}^{\mathrm{LP}}=1-\varepsilon^{2} / 16+17 \varepsilon^{4} / 3072$, respectively. The attained approximation agrees well with the 4 th-order LP solution. Table 1 indicates that $\omega_{2}^{\mathrm{IS}}$ is more accurate than $\omega_{2}^{\mathrm{LP}}$ when compared with $\omega_{4}^{\mathrm{LP}}$.

Figure 1 shows the comparison of the phase planes between iteration solutions $\left(x_{k}\right)$ and numerical result. Rapid convergence of $x_{k}$ to the numerical result can be observed. Note that all numerical solutions are obtained by the fourthorder Runge-Kutta (RK) integration method. When $|\varepsilon|>1$, the iteration procedure presented by Chen and Liu [16] does not converge. This is probably the difference between the starting function $\left(x_{0}=\alpha_{0} \cos t\right)$ and the exact solution is too large. The modified MIM is still effective for $|\varepsilon| \leq 1.5$. As Figure 2 shows, the LC solution with $\varepsilon=1.5$ obtained by the presented method is in excellent agreement with numerical one. It is necessary to point out that the presented method is able to track unstable LCs, whereas the RK method is not.

Also plotted in Figure 2 are the results provided by the LP method [17]. The iteration results are much more precise than the 2nd and 4th-order LP approximations.

It is necessary to point out that the presented method is able to track unstable LCs, whereas the RK method is not. Figure 3 shows an unstable LC of the van der Pol equation with $\varepsilon=-1$ obtained by the presented method. As shown, the RK begins at the LC; however, the solution curve converges to the equilibrium.

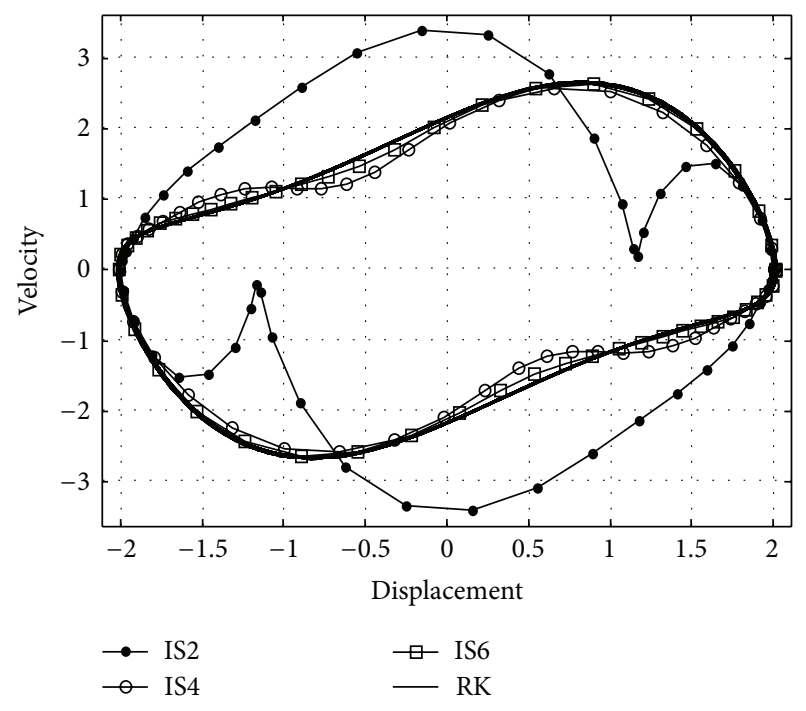

FIGURE 1: The LC solutions of system (10) with $\varepsilon=1$ obtained by the modified MIM and RK method, respectively. The $k$ th-order solution $\left(x_{k}\right)$ is represented by ISk.

In order to further demonstrate the merit of the modified MIM when applied to problems with nonlinear damping terms, we consider the following self-excited system [18]:

$$
\ddot{x}+x+\varepsilon\left[(\dot{x})^{2}-1\right] \dot{x}+f(\dot{x})=0 .
$$

The nonlinear term contains high powers of $\dot{x}$, that is, $f(\dot{x})=$ $(\dot{x})^{3}$. If the original MIM is employed, the algebraic equations governing $\omega$ will become very complicated. Therefore, it is necessary to employ the modified approach. Figure 4 indicates that the approximations obtained by the presented method converge rapidly to the numerical solution as $k$ increased.

Example 2 (system with nonsmooth nonlinearity). The modified MVIM is further applied to nonsmooth dynamical system expressed as

$$
\ddot{x}+f(x, \dot{x})+\eta g(x)=0 .
$$

Here, $f(x, \dot{x})$ is a nonlinear damping term, and $g(x)$ is a nonsmooth function. If substituting the $x_{k-1}$ into $g(x)$, on account of $x_{k-1}$ contained unknown quantities $\left(\alpha_{k-1}\right)$, so $g(x)$ can not be expanded as Fourier progression by numerical integration. To this end, (9) cannot be deduced by eliminating 


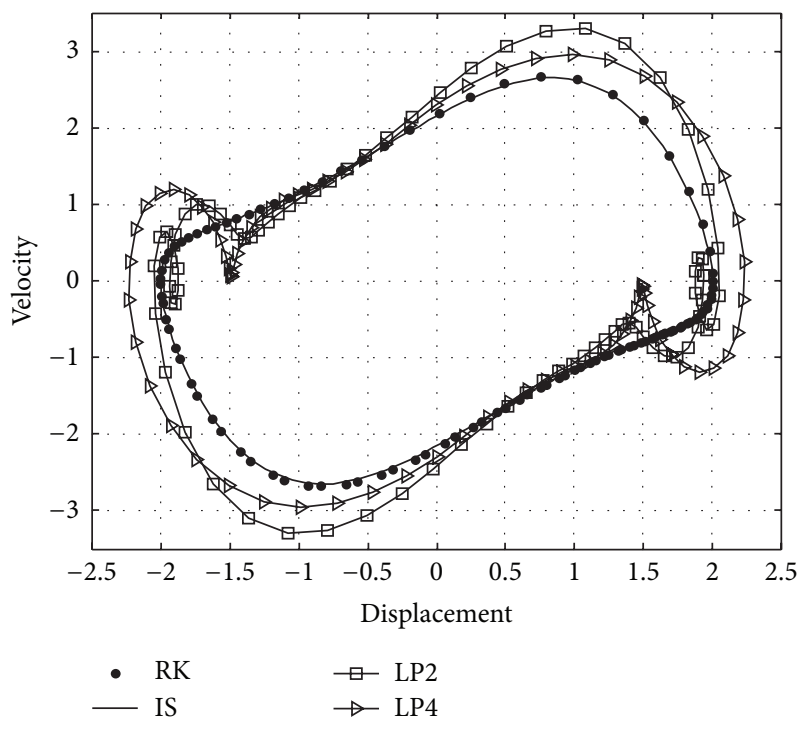

FIGURE 2: The LC solutions of system (10) with $\varepsilon=1.5$ obtained by the modified MIM, RK method, and LP method, respectively. The iteration solution is denoted as IS and the $k$ th-order LP approximation as LPk.

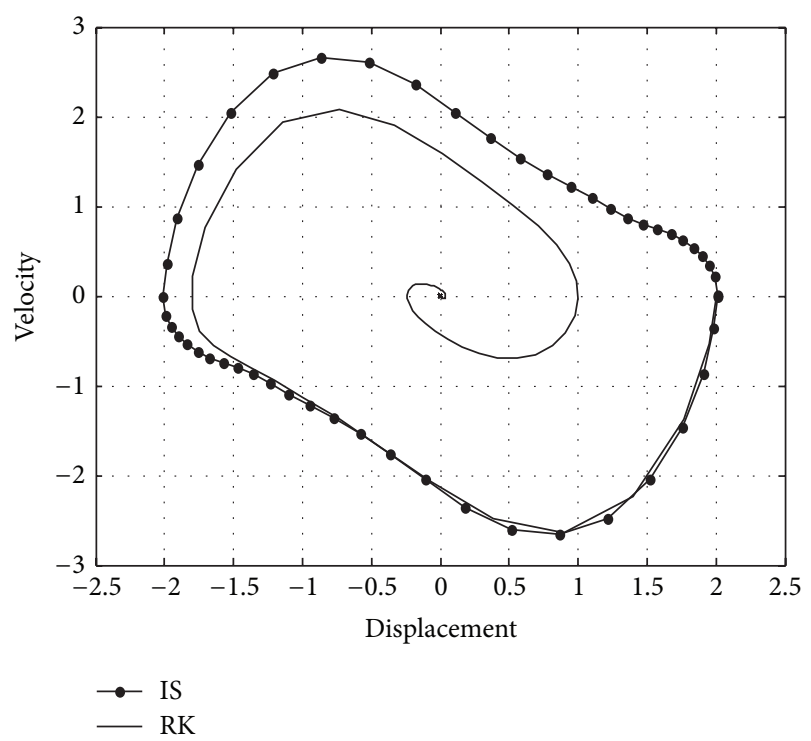

FIGURE 3: Comparison of the LC solution of system (10) with $\varepsilon=-1$, provided by the modified MIM, and by RK method, respectively.

the secular terms. Likewise, we present the following iteration scheme:

$$
\omega_{k-1}^{2}\left(\ddot{x}_{k}+x_{k}\right)=\omega_{k-1}^{2} x_{k-1}+f\left(x_{k-1}, \omega_{k-2} \dot{x}_{k-1}\right)+\eta g\left(x_{k-2}\right) .
$$

In this scheme, $g\left(x_{k-2}\right)$ can be expanded as a Fourier series since $\alpha_{k-2}$ has been determined at the previous iteration.

Let us consider a van der Pol type oscillator with a nonsmooth function as

$$
\ddot{x}+x+\varepsilon\left(1-x^{2}\right) \dot{x}+\eta g(x)=0
$$

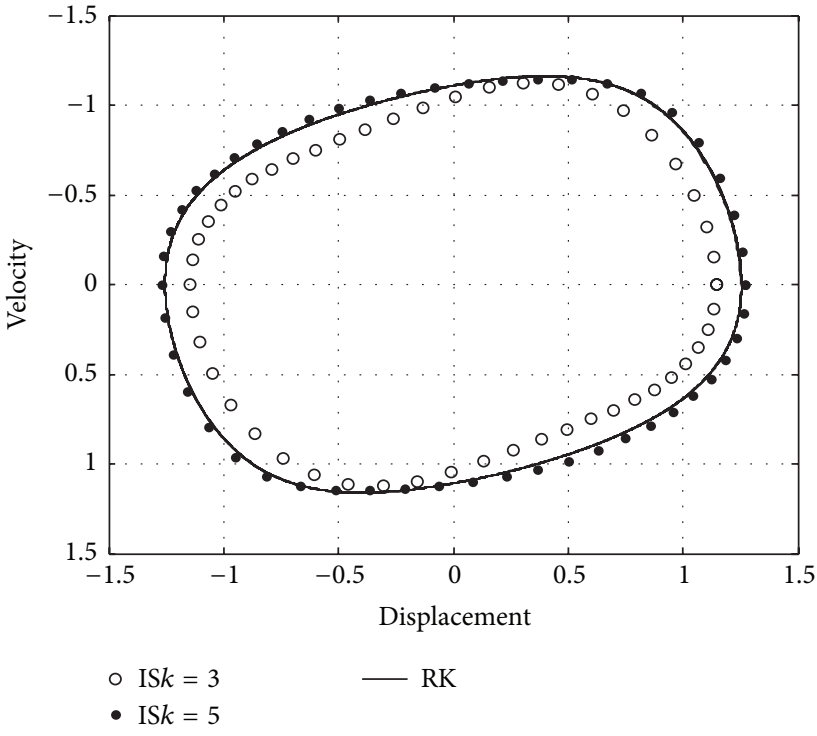

FIgURE 4: The LC solutions of system (21) with $\varepsilon=1$ obtained by the modified MIM and RK method, respectively. The $k$ th-order solution $\left(x_{k}\right)$ is represented by ISk.

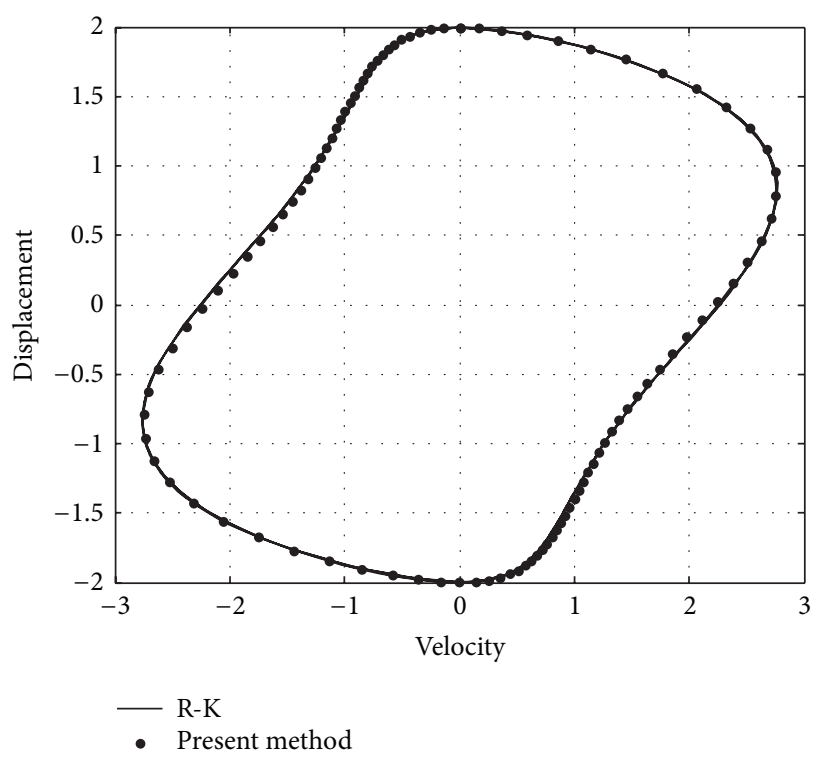

FIGURE 5: LC solutions of system (24) with (25) $(\varepsilon=1, \eta=0.5)$ provided by the modified MIM and by RK method, respectively.

with

$$
g(x)= \begin{cases}x-1 & x \geq 1 \\ 0 & -1<x<1 \\ x+1 & x \leq-1\end{cases}
$$

Figure 5 shows the LC of system (14) with $\varepsilon=1$ and $\eta=$ 0.5 . The 5 th-order approximations obtained by the presented 


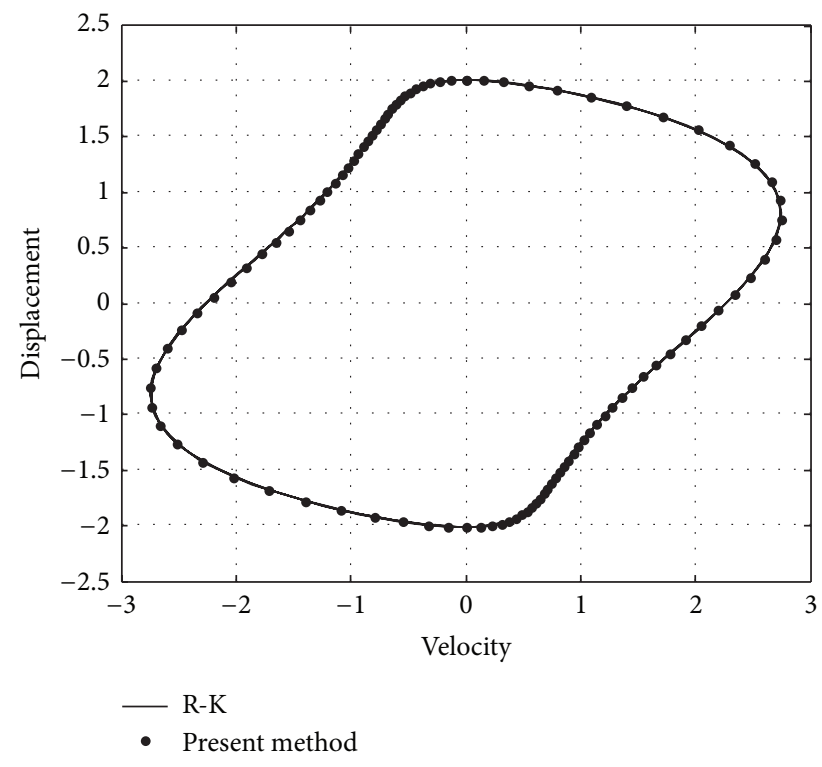

FIGURE 6: LC solutions of system (24) with (26) $(\varepsilon=1, \eta=0.5)$ provided by the modified MIM and by RK method, respectively.

method agree well with the numerical solution when the nonsmooth term is given as

$$
g(x)=\operatorname{sgn}(x)= \begin{cases}1, & x>0 \\ 0, & x=0 \\ -1, & x<0\end{cases}
$$

The LC can also be obtained very accurate, as Figure 6 shows.

\section{Conclusions}

The Mickens iteration method (MIM) has been modified, so that it is suitable for solving LC solutions of self-excited systems with nonsmooth and/or damping nonlinearities. Different from the routinely-used MIM, the modified method decouples the unknown frequency from nonlinear terms. This modification simplifies the MIM significantly. Numerical examples show the feasibility and validity of the presented method, which implies that it could be applicable to more nonlinear dynamical systems, especially those with nonlinear damping terms and nonsmooth nonlinearities.

\section{Acknowledgment}

This work is supported by the National Natural Science Foundation of China (11002088, 11272361, 11172333), Doctoral Program Foundation of Ministry of Education of China (20130171110039), Guangdong Province Natural Science Foundation (S2012040007920, S2013010013802), Fundamental Research Funds for the Central Universities (13lgzd06), and the Guangdong Province Science and Technology Program (2012A030200011).

\section{References}

[1] R. E. Mickens, "Iteration procedure for determining approximate solutions to nonlinear oscillator equations," Journal of Sound and Vibration, vol. 116, no. 1, pp. 185-187, 1987.

[2] R. E. Mickens, "Harmonic balance and iteration calculations of periodic solutions to $y^{\prime \prime}+y^{-1}=0$," Journal of Sound and Vibration, vol. 306, no. 3-5, pp. 968-972, 2007.

[3] R. E. Mickens, "A generalized iteration procedure for calculating approximations to periodic solutions of "truly nonlinear oscillators'”' Journal of Sound and Vibration, vol. 287, no. 4-5, pp. 10451051, 2005.

[4] S. Bhattacharjee and J. K. Bhattacharjee, "Lindstedt Poincare technique applied to molecular potentials," Journal of Mathematical Chemistry, vol. 50, no. 6, pp. 1398-1410, 2012.

[5] H. Hu and J. H. Tang, "A classical iteration procedure valid for certain strongly nonlinear oscillators," Journal of Sound and Vibration, vol. 299, no. 1-2, pp. 397-402, 2007.

[6] J. I. Ramos, "On Linstedt-Poincaré technique for the quintic Duffing equation," Applied Mathematics and Computation, vol. 193, no. 2, pp. 303-310, 2007.

[7] F. K. Yin, J. Q. Song, and X. Q. Cao, "Couple of the variational iteration method and Legendre wavelets for nonlinear partial differential equations," Journal of Applied Mathematics, vol. 2013, Article ID 157956, 11 pages, 2013.

[8] M. T. Atay and O. Kilic, “The semianalytical solutions for stiff systems of ordinary differential equations by using variational iteration method and modified variational iteration method with comparison to exact solutions," Mathematical Problems in Engineering, vol. 2013, Article ID 143915, 11 pages, 2013.

[9] A.-J. Chen, "Resonance analysis for tilted support spring coupled nonlinear packaging system applying variational iteration method," Mathematical Problems in Engineering, vol. 2013, Article ID 384251, 4 pages, 2013.

[10] V. Marinca, N. Herişanu, and C. Bota, "Application of the variational iteration method to some nonlinear one-dimensional oscillations," Meccanica, vol. 43, no. 1, pp. 75-79, 2008.

[11] C. W. Lim and B. S. Wu, "A modified Mickens procedure for certain non-linear oscillators," Journal of Sound and Vibration, vol. 257, no. 1, pp. 202-206, 2002.

[12] V. Marinca and N. Herisanu, "A modified iteration perturbation method for some nonlinear oscillation problems," Acta Mechanica, vol. 184, no. 1-4, pp. 231-242, 2006.

[13] H. Hu, "Solutions of the Duffing-harmonic oscillator by an iteration procedure," Journal of Sound and Vibration, vol. 298, no. 1-2, pp. 446-452, 2006.

[14] Y. M. Chen, G. Meng, and J. K. Liu, "A new method for Fourier series expansions: applications in rotor-seal systems," Mechanics Research Communications, vol. 38, no. 5, pp. 399-403, 2011.

[15] T. Pirbodaghi, M. T. Ahmadian, and M. Fesanghary, "On the homotopy analysis method for non-linear vibration of beams," Mechanics Research Communications, vol. 36, no. 2, pp. 143-148, 2009.

[16] Y. M. Chen and J. K. Liu, "A modified Mickens iteration procedure for nonlinear oscillators," Journal of Sound and Vibration, vol. 314, no. 3-5, pp. 465-473, 2008.

[17] A. H. Nayfeh, Introduction to Perturbation Techniques, WileyInterscience, New York, NY, USA, 1993.

[18] W. J. Ding, Self-Excited Vibration, Tsinghua University Press, Beijing, China, 2009. 


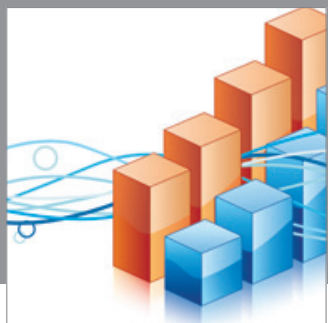

Advances in

Operations Research

mansans

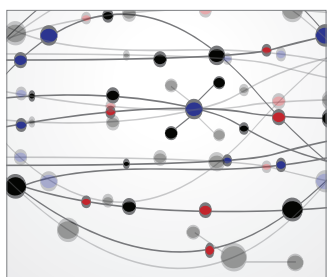

The Scientific World Journal
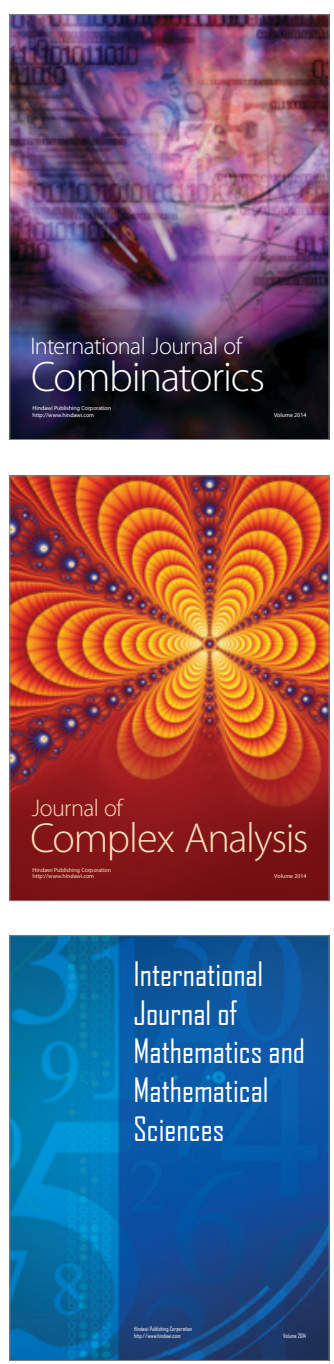
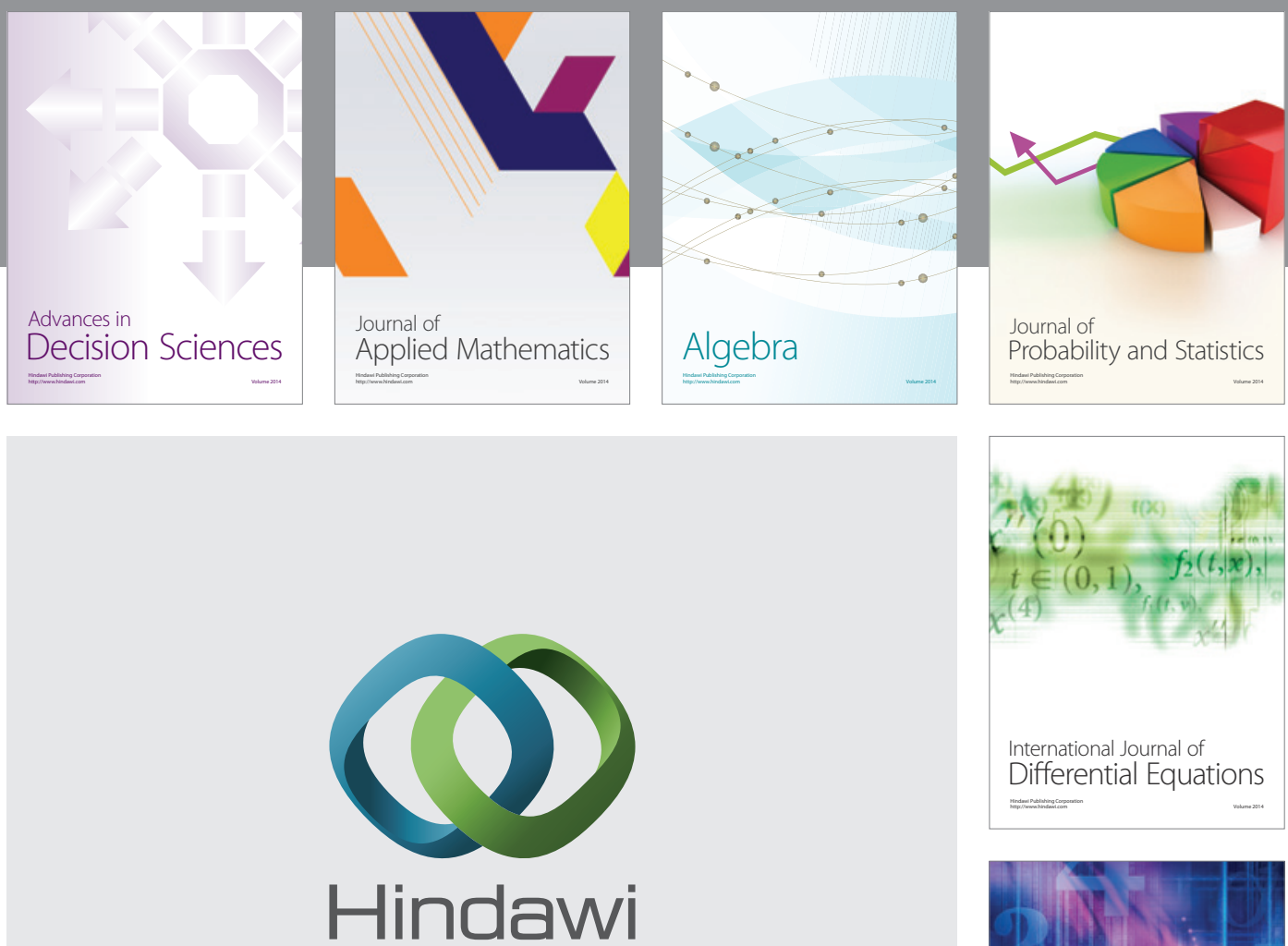

Submit your manuscripts at http://www.hindawi.com
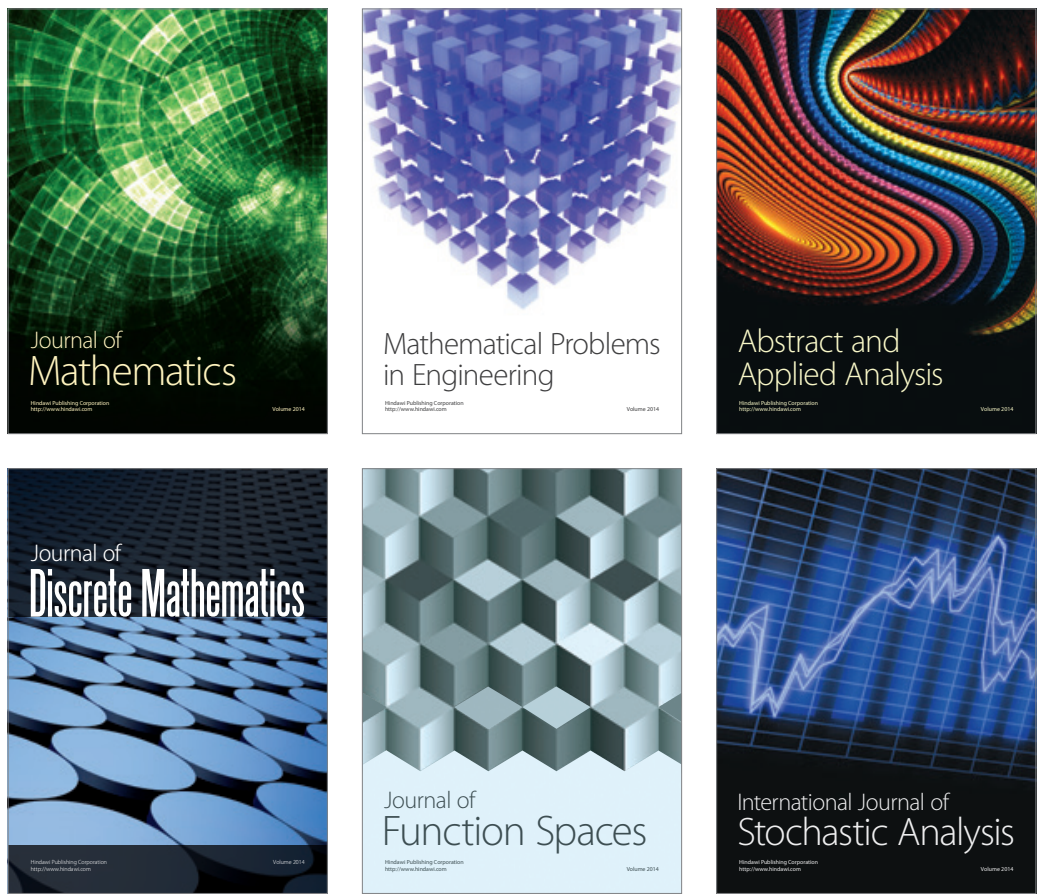

Journal of

Function Spaces

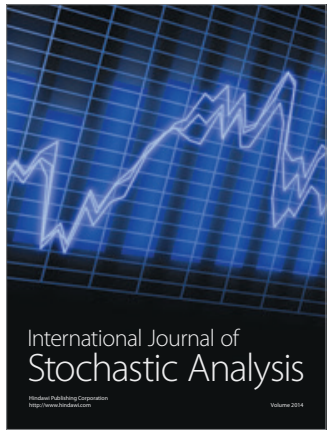

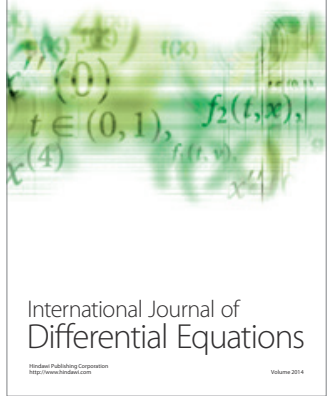
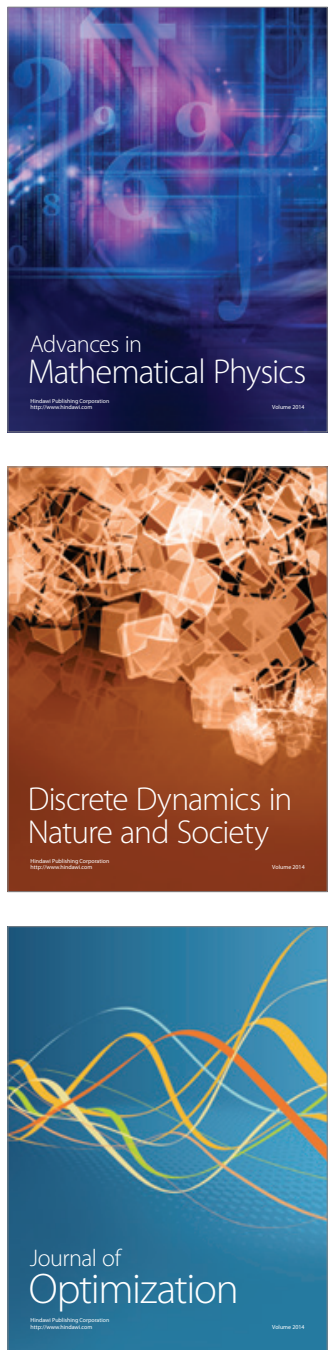